\title{
Generalized obstacle problem
}

\author{
By
}

Rie SASAI

\begin{abstract}
In their 1995 paper [3], Fehlmann and Gardiner posed an extremal problem, which will be called an obstacle problem throughout the present paper, for quadratic differentials on Riemann surfaces. A compact set $E$ in a Riemann surface $S$ of finite type is called an obstacle if each component of $E$ is relatively contractible in $S$ and if $S \backslash E$ is connected. For a given obstacle $E$ and a symmetric integrable quadratic differential $\varphi \neq 0$ on $S$, the obstacle problem is to find a conformal embedding $g$ of $S \backslash$ $E$ into another Riemann surface $R$ of the same type as $S$ and a symmetric quadratic differential $\psi$ on $R$ so that the following three conditions hold: (i) the borders and punctures are preserved under the mapping $g$, (ii) the pull-back $g^{*} \psi$ gives the same heights vector as that of $\varphi$, and (iii) the norm $\|\psi\|_{L^{1}(R)}$ is maximal among those embeddings. Fehlmann and Gardiner asserted existence and uniqueness of a solution to the obstacle problem when $E$ consists only of finitely many components. It seems, however, that the uniqueness assertion is not correct in their form. In the present paper, we extend the existence theorem and give a correction to the uniqueness assertion in the general case. As an application we provide a slit mapping theorem for an open Riemann surface of finite genus.
\end{abstract}

\section{Introduction}

Since early 20th century, through the work of Koebe, de Possel and others, it has turned out that a solution to an extremal problem often gives a conformal mapping with distinguished properties, such as parallel slit mappings. In this regard, it is important to find a good extremal problem for the investigation of complex analysis. Fehlmann and Gardiner [3] studied such an extremal problem, which will be called the obstacle problem (see also [5, Chap.13]). In this paper, we extend their results to the case where an obstacle may have uncountably many connected components. In order to formulate the problem, we give some explanations for necessary notions.

1991 Mathematics Subject Classification(s). Primary 30F60; Secondary 30C75, 32G15

Received September 6, 2004

Revised November 5, 2004 
For a holomorphic quadratic differential $\varphi=\varphi(z) d z^{2}$ on a Riemann surface $S$, we define the $L^{1}$-norm $\|\varphi\|_{L^{1}(S)}$ of it by

$$
\|\varphi\|_{L^{1}(S)}=\iint_{S}|\varphi(z)| d x d y, \quad z=x+i y .
$$

If $\|\varphi\|_{L^{1}(S)}<\infty$, the quadratic differential $\varphi$ is called integrable. We denote by $A(S)$ the set of integrable holomorphic quadratic differentials on $S$.

Suppose that $S$ is a hyperbolic Riemann surface. Then there is a holomorphic universal covering projection $P$ of $\mathbb{D}=\{z \in \mathbb{C}:|z|<1\}$ onto $S$. The group

$$
\Gamma=\{\gamma \in \operatorname{AutD}: P \circ \gamma=P\}
$$

is called a Fuchsian model of $S$. When $\Gamma$ is of the second kind, namely, when the limit set $\Lambda(\Gamma)$ of $\Gamma$ does not coincide with $\partial \mathbb{D}$, the Riemann surface $S^{\text {d }}=$ $(\overline{\mathbb{C}} \backslash \Lambda(\Gamma)) / \Gamma$ is called the (Schottky) double of $S$, and $\partial S=(\partial \mathbb{D} \backslash \Lambda(\Gamma)) / \Gamma$ is called the border of $S$. When $\Gamma$ is of the first kind, we set $S^{\mathrm{d}}=S$ and $\partial S=\emptyset$.

Let $S$ be a Riemann surface of finite type $(\kappa, m, l)$, that is, $S$ is obtained by removing mutually disjoint $l$ topological compact disks and $m$ points from a compact Riemann surface of genus $\kappa$. When $l=0$, we say that $S$ is of finite analytic type $(\kappa, m)$. When $l>0$, the double $S^{\mathrm{d}}$ is of finite analytic type $(2 \kappa+l-1,2 m)$. We assume that the number $6 \kappa-6+3 l+2 m$ is positive in the sequel so that $\operatorname{dim}_{\mathbb{C}} A\left(S^{\mathrm{d}}\right)>0$.

A quadratic differential $\varphi=\varphi(z) d z^{2}$ in $A(S)$ is called symmetric relative to $\partial S$ if there is a quadratic differential $\varphi^{\mathrm{d}} \in A\left(S^{\mathrm{d}}\right)$ with $\left.\varphi^{\mathrm{d}}\right|_{S}=\varphi$ for which $\overline{j^{*} \varphi^{\mathrm{d}}}=\varphi^{\mathrm{d}}$ holds. Here $j: S^{\mathrm{d}} \rightarrow S^{\mathrm{d}}$ is the canonical anti-conformal involution of $S^{\mathrm{d}}$ induced by the conjugation $z \mapsto 1 / \bar{z}$, in other words, the lift $\tilde{\varphi}$ of $\varphi^{\mathrm{d}}$ to $\overline{\mathbb{C}} \backslash \Lambda(\Gamma)$ is symmetric relative to $\partial \mathbb{D}: \overline{\tilde{\varphi}(1 / \bar{z})} \cdot z^{-4}=\tilde{\varphi}(z)$. We denote by $A_{\mathrm{s}}(S)$ the set of quadratic differentials $\varphi \in A(S)$ symmetric relative to $\partial S$. Note that $\operatorname{dim}_{\mathbb{R}} A_{\mathrm{s}}(S)=6 \kappa-6+3 l+2 m$ if $S$ is of finite type $(\kappa, m, l)$ with $l>0$.

For a Riemann surface $S$ of finite analytic type let $\mathfrak{S}(S)$ be the set of simple closed curves $\gamma$ on $S$ which are homotopic neither to a point in $S$ nor to a puncture of $S$, and let $\mathfrak{S}[S]$ be the set of the free homotopy classes $[\gamma]$ of $\gamma \in \mathfrak{S}(S)$.

For a given $\varphi \in A(S) \backslash\{0\}$ and $\gamma \in \mathfrak{S}(S)$, we set

$$
\operatorname{height}_{\varphi}(\gamma):=\int_{\gamma}|\operatorname{Im}(\sqrt{\varphi(z)} d z)|
$$

and

$$
\operatorname{height}_{\varphi}[\gamma]:=\inf _{\beta} \operatorname{height}_{\varphi}(\beta),
$$

where the infimum is taken over all closed curves $\beta \in \mathfrak{S}(S)$ freely homotopic to $\gamma$.

We are now ready to state the obstacle problem in the sense of Fehlmann and Gardiner [3]. They thought of a "simply connected" compact subset with finitely many connected components as an obstacle. Here we will include a set 
with uncountably many components as an obstacle. In this paper we say that a subset $E$ of a Riemann surface $S$ is relatively contractible in $S$ if there is a continuous map $H: E \times[0,1] \rightarrow S$ such that $H(p, 1)=p$ for all $p \in E$ and that $H(p, 0)=p_{0} \in S$ is independent of $p \in E$.

Definition 1.1 (allowable subset). A subset $E$ of a Riemann surface $S$ is said to be allowable if $E$ is compact and relatively contractible in $S$ and if $S \backslash E$ is connected. For an element $\varphi \in A(S)$, if each component of an allowable subset $E$ is either a horizontal arc (resp. vertical arc) of $\varphi$ or the union of finitely many horizontal arcs (resp. vertical arcs) and critical points of $\varphi$, then $E$ is called an allowable horizontal slit (resp. allowable vertical slit) with respect to $\varphi$.

Note that an allowable set $E$ may have uncountably many components.

Let $S$ be a Riemann surface of finite type and that $E \subset S$ be allowable. A conformal embedding $g: S \backslash E \rightarrow S_{g}$ will be called type-preserving (with respect to $S$ ) if $S_{g}$ is a Riemann surface of the same type as $S$ and if $g$ maps the border and the punctures of $S$ onto the border and the punctures of $S_{g}$, respectively. Then the mapping $g$ naturally extends to a conformal map $g^{\mathrm{d}}$ of $S^{\mathrm{d}} \backslash E^{\mathrm{d}}$ into the double $S_{g}^{\mathrm{d}}$ of $S_{g}$. We remark that $E^{\mathrm{d}}=E \cup j(E)$ and $E_{g}=S_{g} \backslash g(S \backslash E)$ are both allowable (see Lemma 2.2). For further properties of an allowable set, see Section 2. Then $g^{\mathrm{d}}$ induces an isomorphism $\iota_{g}$ of the fundamental group $\pi_{1}\left(S^{\mathrm{d}}\right)$ of $S^{\mathrm{d}}$ onto that of $S_{g}^{\mathrm{d}}$ (see Lemma 2.3). Note that the isomorphism $\iota_{g}$ induces naturally the bijection

$$
\iota_{g}: \mathfrak{S}\left[S^{\mathrm{d}}\right] \rightarrow \mathfrak{S}\left[S_{g}^{\mathrm{d}}\right] .
$$

Definition 1.2 (obstacle problem). Let $E$ be an allowable subset of a Riemann surface $S$ of finite type and $\varphi \in A_{\mathrm{s}}(S) \backslash\{0\}$. The obstacle problem for $(S, E, \varphi)$ is to find a triple $\left(g, S_{g}, \psi\right)$, where $g$ is a type-preserving conformal embedding of $S \backslash E$ with respect to $S$ into another Riemann surface $S_{g}$ and $\psi \in A_{\mathrm{s}}\left(S_{g}\right)$, with the following properties:

(i) height $\psi_{\psi^{\mathrm{d}}} \circ \iota_{g}=$ height $_{\varphi^{\mathrm{d}}}$ on $\mathfrak{S}\left[S^{\mathrm{d}}\right]$ and

(ii) the norm $\|\psi\|_{L^{1}\left(S_{g}\right)}$ is maximal among those triples satisfying (i).

Our first main theorem is stated as in the following, which is a generalization of a result of Fehlmann and Gardiner [3].

Theorem 1.3 (existence). $\quad$ Suppose that $S$ is a Riemann surface of $f$ nite type and that $\varphi \in A_{\mathrm{S}}(S) \backslash\{0\}$. Let $E$ be an allowable subset of $S$. Then there is a solution to the obstacle problem for $(S, E, \varphi)$. For every solution $\left(g, S_{g}, \psi\right), E_{g}=S_{g} \backslash g(S \backslash E)$ is an allowable horizontal slit with respect to $\psi$, and $E_{g}$ is of zero area.

In [3], Fehlmann and Gardiner showed the above result under the additional assumption that $E$ consists of finitely many components. They also asserted in the paper that the solution $\left(g, S_{g}, \psi\right)$ is unique in the sense that, if $\left(u, S_{u}, \theta\right)$ is also a solution for $(S, E, \varphi)$, then $g \circ u^{-1}$ extends to a conformal 
map $w$ of $S_{u}$ onto $S_{g}$ and $w_{*} \theta=\psi$ holds. The uniqueness, however, does not necessarily hold in this sense (see [9] for a counterexample).

In this paper we show a uniqueness result for the obstacle problem in the following form.

Theorem 1.4 (uniqueness). Under the same hypotheses as in Theorem 1.3 , the extremal differential $\psi \in A\left(S_{g}\right) \backslash\{0\}$ is uniquely determined. Namely, if a triple $\left(u, S_{u}, \theta\right)$ is also a solution for $(S, E, \varphi)$, then $\theta$ satisfies

$$
\theta=(\psi \circ w)\left(w^{\prime}\right)^{2} \text { on } u(S \backslash E),
$$

where $w=g \circ u^{-1}$.

The present paper is organized as follows. Section 2 will be devoted to preparations including basic properties of allowable sets and some facts about quadratic differentials. Theorems 1.3 and 1.4 will be proved in Sections 3 and 4 , respectively. In Section 5, as an application, we give a slit mapping theorem for an open Riemann surface of finite genus.

We remark that, if we consider the extremal problem of finding a triple $\left(g, S_{g}, \psi\right)$ satisfying (i) in Definition 1.2 and

(ii)' the norm $\|\psi\|_{L^{1}\left(S_{g}\right)}$ is minimal among those triples satisfying (i), then the similar result can be obtained just by replacing the term "horizontal" by "vertical". That is, there is a solution of the extremal problem and for every solution $\left(g, S_{g}, \psi\right), E_{g}$ is an allowable vertical slit with respect to $\psi$, and $E_{g}$ is of zero area.

\section{Preliminaries}

We collect here basic properties of allowable subsets of a Riemann surface. First we give another characterization of relatively contractible set in $S$.

Lemma 2.1. Let $E$ be a compact subset of a Riemann surface $S$. Then $E$ is relatively contractible in $S$ if and only if $E$ is contained in a compact topological disk in $S$.

Proof. The "if" part is trivial. We show the "only if" part when $S$ is hyperbolic. The non-hyperbolic cases can be treated similarly. Suppose that $E$ is relatively contractible in $S$. Then there is a continuous map $H$ : $E \times[0,1] \rightarrow S$ such that $H(p, 1)=p$ for all $p \in E$ and that $H(p, 0)=p_{0}$ is independent of $p \in E$. Let $P$ be the universal covering projection of $\mathbb{D}$ onto $S$ such that $P(0)=p_{0}$ and let $\Gamma$ be the covering transformation group of $P$. Let $\tilde{H}: E \times[0,1] \rightarrow \mathbb{D}$ be a lift of the homotopy $H$ via $P$ such that $\tilde{H}(p, 0)=0$ for all $p \in E$. The set $\tilde{E}=\{\tilde{H}(p, 1): p \in E\}$ is compact. For every $\tilde{p} \in \tilde{E}$ we can take a positive number $r_{\tilde{p}}$ with the property that the closed disk $V_{\tilde{p}}$ centered at $\tilde{p}$ with radius $r_{\tilde{p}}$ satisfies

$$
\operatorname{dist}\left(A\left(V_{\tilde{p}}\right), \tilde{E}\right) \geq 2 r_{\tilde{p}} \text { for every } A \in \Gamma, A \neq \mathrm{id} .
$$


By compactness of $\tilde{E}$ we may assume that there exist finitely many points $\tilde{p}_{1}, \ldots, \tilde{p}_{k} \in \tilde{E}$ so that $\tilde{E}$ is contained in the interior of the closed set $\tilde{V}=$ $\cup_{i=1}^{k} V_{\tilde{p}_{i}}$. We may assume that by replacing radii, if necessary, $V_{\tilde{p}_{i}}$ is not tangent to $V_{\tilde{p}_{j}}$ for every $i \neq j$. We can see that $A(\tilde{V}) \cap \tilde{V}=\emptyset$ for every $A \in \Gamma, A \neq \mathrm{id}$. Indeed, if $A(\tilde{V}) \cap \tilde{V} \neq \emptyset$ for some $A \in \Gamma, A \neq \mathrm{id}$, there exist $i, j \in\{1, \ldots, k\}$ and $\tilde{q}_{i} \in V_{\tilde{p}_{i}}$ and $\tilde{q}_{j} \in V_{\tilde{p}_{j}}$ such that $A\left(\tilde{q}_{i}\right)=\tilde{q}_{j}$. Because of $\operatorname{dist}\left(A\left(V_{\tilde{p}_{i}}\right), \tilde{E}\right) \geq 2 r_{\tilde{p}_{i}}$, we have $r_{\tilde{p}_{j}} \geq \operatorname{dist}\left(\tilde{q}_{j}, \tilde{p}_{j}\right) \geq \operatorname{dist}\left(\tilde{q}_{j}, \tilde{E}\right) \geq \operatorname{dist}\left(A\left(V_{\tilde{p}_{i}}\right), \tilde{E}\right) \geq 2 r_{\tilde{p}_{i}}$ holds. On the other hand, by considering $A^{-1}$, we have $r_{\tilde{p}_{i}} \geq 2 r_{\tilde{p}_{j}}$. This is a contradiction. Hence $A(\tilde{V}) \cap \tilde{V}=\emptyset$ for every $A \in \Gamma, A \neq$ id. Let $\tilde{W}$ be a polynomially convex hull of $\tilde{V}$, that is $\tilde{W} \subset \mathbb{D}$ is the union of $\tilde{V}$ and all compact components of $\mathbb{D} \backslash \tilde{V}$. Then $\tilde{W}$ is a disjoint union of finitely many compact topological disks whose boundaries are contained in $\tilde{V}$. Then we can see that for every components $\tilde{W}_{1}, \tilde{W}_{2}$ of $\tilde{W}$ and for every $A \in \Gamma, A \neq \mathrm{id}$, the intersection $A\left(\tilde{W}_{1}\right) \cap \tilde{W}_{2}$ is empty or coincident with either $A\left(\tilde{W}_{1}\right)$ or $\tilde{W}_{2}$, because by the above argument $A\left(\partial \tilde{W}_{1}\right) \cap \partial \tilde{W}_{2}=\phi$ holds. Therefore the projection $P(\tilde{W})$ also consists of mutually disjoint compact topological disks in $S$ whose union contains $E$ in its interior. Consequently, by joining these disks with suitable canals we obtain a compact topological disk in $S$ containing $E$.

By using the above lemma, we can also show the following.

Lemma 2.2. Let $E$ be an allowable subset of a Riemann surface $S$ of finite type. Then $E^{\mathrm{d}}=E \cup j(E)$ is an allowable subset of the double $S^{\mathrm{d}}$. Also, for any type-preserving conformal embedding $g: S \backslash E \rightarrow S_{g}$ with respect to $S$ the set $E_{g}=S_{g} \backslash g(S \backslash E)$ is allowable in $S_{g}$.

Proof. By joining a compact topological disk $\Delta$ containing $E$ and its reflection in $\partial S$ with a suitable canal, we obtain a compact topological disk $\Delta^{\mathrm{d}}$ containing $E^{\mathrm{d}}$. Thus $E^{\mathrm{d}}$ is allowable by Lemma 2.1. We now show the latter assertion. We may take a compact topological disk $\Delta$ so that $E$ is contained in the interior of $\Delta$. If $S$ is of finite type $(\kappa, m, l)$, then $S \backslash \Delta$ and $g(S \backslash \Delta)$ are of type $(\kappa, m, l+1)$. Then the set $\Delta_{g}=S_{g} \backslash g(S \backslash \Delta) \subset S_{g}$ is of finite type, say, $\left(\kappa^{\prime}, m^{\prime}, l^{\prime}\right)$. Since $S_{g}$ is reconstructed from $g(S \backslash \Delta)$ and $\Delta_{g}$ by gluing along a single Jordan curve, $(\kappa, m, l)=(\kappa, m, l+1)+\left(\kappa^{\prime}, m^{\prime}, l^{\prime}\right)-(0,0,2)$, i.e., $\left(\kappa^{\prime}, m^{\prime}, l^{\prime}\right)=(0,0,1)$. Then the set $\Delta_{g}$ which contains $E_{g}$ in the interior is a compact topological disk in $S_{g}$. Thus the latter assertion has been proved.

Lemma 2.3. Let $E$ be an allowable subset of a Riemann surface $S$ of finite type. Then the induced homomorphism $\sigma: \pi_{1}(S \backslash E) \rightarrow \pi_{1}(S)$ by the inclusion mapping $S \backslash E \hookrightarrow S$ is surjective. Furthermore, let $g: S \backslash E \rightarrow S_{g}$ be a type-preserving conformal embedding with respect to $S$. Then the induced isomorphism $(g)_{*}: \pi_{1}(S \backslash E) \rightarrow \pi_{1}\left(S_{g} \backslash E_{g}\right)$ decends to an isomorphism $\overline{(g)_{*}}$ : $\pi_{1}(S) \rightarrow \pi_{1}\left(S_{g}\right)$ so that the following diagram commutes, where $\sigma_{g}: \pi_{1}\left(S_{g} \backslash\right.$ 
$\left.E_{g}\right) \rightarrow \pi_{1}\left(S_{g}\right)$ is the induced homomorphism:

$$
\begin{array}{ccc}
\pi_{1}(S \backslash E) & \stackrel{(g)_{*}}{\longrightarrow} & \pi_{1}\left(S_{g} \backslash E_{g}\right) \\
\sigma \downarrow & & \sigma_{g} \downarrow \\
\pi_{1}(S) & \stackrel{(g)_{*}}{\longrightarrow} & \pi_{1}\left(S_{g}\right) .
\end{array}
$$

Proof. By Lemma 2.2 there is a compact topological disk $V \subset S$ bounded by an analytic curve and containing $E$ in its interior $V^{\circ}$. Let $\gamma$ be an arbitrary closed curve in $S$. We will find a closed curve $\gamma^{\prime}$ in $S \backslash V^{\circ}$ freely homotopic to $\gamma$ in $S$. We may assume that $\gamma$ is smooth and intersects $\partial V$ transversally at finitely many points. If $\gamma \cap \partial V=\emptyset$, we can take $\gamma^{\prime}$ as $\gamma$ itself. If $\gamma \cap \partial V \neq \emptyset$, $\gamma$ can be devided into closed subarcs $\gamma_{1}, \delta_{1}, \gamma_{2}, \delta_{2}, \ldots, \gamma_{k}, \delta_{k}$ in such a way that $\gamma=\gamma_{1} \delta_{1} \gamma_{2} \delta_{2} \cdots \gamma_{k} \delta_{k}, \gamma_{j} \subset S \backslash V^{\circ}$ and $\delta_{j} \subset V$ for $j=1,2, \ldots, k$. Let $\delta_{j}^{\prime}$ be a subarc of $\partial V$ sharing end points with $\delta_{j}$. Set $\gamma^{\prime}=\gamma_{1} \delta_{1}^{\prime} \gamma_{2} \delta_{2}^{\prime} \cdots \gamma_{k} \delta_{k}^{\prime}$. Since $\delta_{j} \sim \delta_{j}^{\prime}$ in $V, \gamma^{\prime} \sim \gamma$ in $S$. Thus we can see that the homomorphism $\sigma: \pi_{1}(S \backslash E) \rightarrow \pi_{1}(S)$ is surjective.

Next define $\overline{(g)_{*}}: \pi_{1}(S) \rightarrow \pi_{1}\left(S_{g}\right)$ by $\overline{(g)_{*}}[\gamma]=\left[g\left(\gamma^{\prime}\right)\right]$, where $\gamma^{\prime}$ is the curve constructed above. By construction, $\overline{(g)_{*}}$ is well-defined, homomorphic and makes the diagram in the lemma commute. Considering $g^{-1}: S_{g} \backslash E_{g} \rightarrow$ $S \backslash E$, we see that $\overline{(g)_{*}}$ is isomorphic.

From Lemmas 2.2 and 2.3 we can see that for every type-preserving conformal embedding $g: S \backslash E \rightarrow S_{g}$ with respect to $S$, the natural isomorphism $\left(g^{\mathrm{d}}\right)_{*}: \pi_{1}\left(S^{\mathrm{d}} \backslash E^{\mathrm{d}}\right) \rightarrow \pi_{1}\left(S_{g}^{\mathrm{d}} \backslash E_{g}^{\mathrm{d}}\right)$ induces an isomorphism $\overline{\left(g^{\mathrm{d}}\right)_{*}}: \pi_{1}\left(S^{\mathrm{d}}\right) \rightarrow$ $\pi_{1}\left(S_{g}^{\mathrm{d}}\right)$. We denote $\overline{\left(g^{\mathrm{d}}\right)_{*}}$ by $\iota_{g}$.

Here we recall a definition of the Teichmüller space $T(S)$ of a Riemann surface $S$ of finite analytic type (see [6] for the compact case). Let $(R, \iota)$ be a marked Riemann surface of the same type as $S$, that is, a pair of a Riemann surface $R$ of the same type as $S$ and an orientation-preserving isomorphism $\iota$ of the fundamental group $\pi_{1}(S)$ onto $\pi_{1}(R)$. (More rigorously, we should consider the fundamental group with base point. Though we do not refer to the base point to avoid complexity, the reader can formulate it in an obvious way.) Two pairs $\left(R_{1}, \iota_{1}\right)$ and $\left(R_{2}, \iota_{2}\right)$ are called (Teichmüller) equivalent if there exists a conformal mapping $u$ of $R_{1}$ onto $R_{2}$ such that $(u)_{*} \circ \iota_{1}=\iota_{2}$. The set of such equivalent classes $[R, \iota]$ is called the Teichmüller space of $S$ and denoted by $T(S)$. Every point in $T(S)$ is represented as $\left[R, f_{*}\right]$ by a (smooth) quasiconformal map $f: S \rightarrow R$. For the existence of such a quasiconformal map, see [7].

We next recall heights mappings (cf. $[4, \S 11.7],[5, \S 12.6])$. Let $|d v|$ be a measured foliation on a Riemann surface $S$ of finite analytic type, namely, there are finitely many singularities $p_{1}, \ldots, p_{t}$ in $\hat{S}$, where $\hat{S}$ is the completion of $S$ and an open cover $\left\{U_{j}\right\}$ of $S \backslash\left\{p_{1}, \ldots, p_{t}\right\}$ and real-valued continuous functions $v_{j}$ on $U_{j}$ with locally $L^{2}$ derivatives in such a way that $v_{j}= \pm v_{k}+$ const. on $U_{j} \cap U_{k}$ and $|d v|$ behaves around each $p_{s}$ like the pull-back of $\left|\operatorname{Im}\left(z^{n / 2} d z\right)\right|$ under a quasiconformal map with certain restriction on the integer $n$. 
Let $\varphi$ be a non-zero integrable holomorphic quadratic differential on a Riemann surface $S$ of finite analytic type. We say that a curve $\gamma \in \mathfrak{S}(S)$ is $\varphi$-polygonal if $\gamma$ is a union of finitely many horizontal and vertical $\operatorname{arcs}$ of $\varphi$. For a measured foliation $|d v|$ on $S$, by abuse of language, we define the height of $\gamma$ relative to $|d v|$ by

$$
\operatorname{height}_{v}(\gamma)=\int_{\gamma}|d v| \text {. }
$$

Note that the height is defined for almost every $\varphi$-polygonal curves in $\mathfrak{S}(S)$. We denote by height $_{v}[\gamma]$ the essential infimum of height ${ }_{v}\left(\gamma^{\prime}\right)$ over $\gamma^{\prime} \in[\gamma]$. Measured foliations $\left|d v_{1}\right|$ and $\left|d v_{2}\right|$ on $S$ are called measure equivalent if height ${ }_{v_{1}}[\gamma]=$ height $_{v_{2}}[\gamma]$ holds for all $[\gamma] \in \mathfrak{S}[S]$. Let $\mathfrak{M F}(S)$ be the set of measure equivalence classes of measured foliations on $S$. Every measure equivalence class $[|d v|] \in$ $\mathfrak{M F}(S)$ induces the real-valued function

$$
\operatorname{height}_{v}: \mathfrak{S}[S] \rightarrow \mathbb{R} \quad\left([\gamma] \mapsto \operatorname{height}_{v}[\gamma]\right) .
$$

In this way, we obtain an embedding

$$
\mathfrak{M F}(S) \rightarrow \mathbb{R}^{\mathfrak{S}[S]}\left([|d v|] \mapsto \text { height }_{v}\right) .
$$

Then the product topology of $\mathbb{R}^{\mathfrak{S}[S]}$ induces a topology of $\mathfrak{M F}(S)$. It is known that the mapping

$$
\Psi: A(S) \backslash\{0\} \rightarrow \mathfrak{M F}(S) \quad(\varphi \mapsto[|\operatorname{Im}(\sqrt{\varphi(z)} d z)|])
$$

is a homeomorphism (see, for example, [5, p. 227]).

For a given $\varphi \in A(S) \backslash\{0\}$ and a local homeomorphism $f: S \rightarrow R$, the measured foliation $|d v|=|\operatorname{Im}(\sqrt{\varphi(z)} d z)|$ on $S$ induces a measured foliation $f_{*}(|d v|)=\left|d\left(v \circ f^{-1}\right)\right|$ on $R$. The relation

$$
\operatorname{height}_{\varphi}[\gamma]=\operatorname{height}_{f_{*}(|d v|)}[f(\gamma)], \quad[\gamma] \in \mathfrak{S}[S],
$$

implies that the measure equivalence class $\left[f_{*}(|d v|)\right] \in \mathfrak{M F}(R)$ depends only on the Teichmüller equivalence class $\tau=\left[R, f_{*}\right]$. Then we obtain the unique holomorphic quadratic differential $\tau_{*} \varphi \in A(R) \backslash\{0\}$ as $\Psi^{-1}\left[f_{*}(|d v|)\right]$, namely,

$$
\begin{aligned}
\operatorname{height}_{\tau_{*} \varphi}[\gamma] & =\operatorname{height}_{f_{*}(|d v|)}[\gamma] \\
& =\operatorname{height}_{\varphi}\left[f^{-1}(\gamma)\right]
\end{aligned}
$$

for every $[\gamma] \in \mathfrak{S}[R]$. The mapping $\tau_{*}: A(S) \backslash\{0\} \rightarrow A(R) \backslash\{0\}$ is called the heights mapping.

The following variant of the second minimal norm property will play an important role in this note. The assertion can be seen by analyzing the proof of Theorem 9 in [4, p. 54].

Proposition 2.4. Let $S$ be a Riemann surface of finite analytic type. Suppose that $\varphi \in A(S)$ and $\psi$ is an integrable (not necessarily holomorphic) quadratic differential on $S$ such that

$$
\operatorname{height}_{\varphi}[\gamma] \leq \operatorname{height}_{\psi}(\gamma)
$$


for almost every $\varphi$-polygonal curve $\gamma \in \mathfrak{S}(S)$. Then

$$
\|\varphi\|_{L^{1}(S)} \leq \iint_{S}|\sqrt{\varphi} \sqrt{\psi}|
$$

and, in particular, $\|\varphi\|_{L^{1}(S)} \leq\|\psi\|_{L^{1}(S)}$. Moreover $\|\varphi\|_{L^{1}(S)}=\|\psi\|_{L^{1}(S)}$ only if $\varphi=\psi$ a.e.

Now we can show

Lemma 2.5. Let $E$ be an allowable subset of a Riemann surface $S$ of finite type and $\varphi \in A_{\mathrm{S}}(S) \backslash\{0\}$. Suppose that $g: S \backslash E \rightarrow S_{g}$ is a type-preserving conformal embedding with respect to $S$. Then there exists the unique $\psi \in A_{\mathrm{s}}\left(S_{g}\right)$ satisfying the condition (i) in Definition 1.2.

Proof. We can take $\psi=\left.\tau_{*}\left(\varphi^{\mathrm{d}}\right)\right|_{S_{g}}$, where $\tau=\left[S_{g}^{\mathrm{d}}, \iota_{g}\right] \in T\left(S^{\mathrm{d}}\right)$ and $\tau_{*}$ is the heights mapping. Then we have only to show that the quadratic differential $\tau_{*}\left(\varphi^{\mathrm{d}}\right)$ is symmetric relative to $\partial S_{g}$. Indeed,

$$
\begin{aligned}
\text { height }_{j_{g}^{*} \tau_{*} \varphi^{\mathrm{d}}}[\gamma] & =\text { height }_{\tau_{*} \varphi^{\mathrm{d}}}\left[j_{g}(\gamma)\right] \\
& =\operatorname{height}_{\varphi^{\mathrm{d}}}\left[\iota_{g}^{-1} \circ j_{g}(\gamma)\right] \\
& =\operatorname{height}_{\varphi^{\mathrm{d}}}\left[j \circ \iota_{g}^{-1}(\gamma)\right] \\
& =\operatorname{height}_{\varphi^{\mathrm{d}}}\left[\iota_{g}^{-1}(\gamma)\right] \\
& =\operatorname{height}_{\tau_{*} \varphi^{\mathrm{d}}}[\gamma],
\end{aligned}
$$

for every $[\gamma] \in \mathfrak{S}\left[S_{g}^{\mathrm{d}}\right]$, where $j_{g}: S_{g}^{\mathrm{d}} \rightarrow S_{g}^{\mathrm{d}}$ is the canonical anticonformal involution of $S_{g}^{\mathrm{d}}$, and Proposition 2.4 implies

$$
\overline{j_{g}^{*} \tau_{*} \varphi^{\mathrm{d}}}=\tau_{*} \varphi^{\mathrm{d}},
$$

that is, $\tau_{*} \varphi^{\mathrm{d}}$ is symmetric relative to $\partial S_{g}$.

The uniqueness of $\psi$ follows from injectivity of $\tau_{*}$.

The quadratic differential $\psi$ in Lemma 2.5 will be denoted by $\varphi_{g}$ in the sequel for a type-preserving conformal embedding $g: S \backslash E \rightarrow S_{g}$ with respect to $S$. We also write

$$
M_{g}=\left\|\varphi_{g}\right\|_{L^{1}\left(S_{g}\right)}
$$

Following [3], we now introduce a few lemmas. Let $S^{\prime}$ be a subdomain of a Riemann surface $S$. For a simple closed curve $\beta$ on $S^{\prime}$ we denote by $\lambda\left([\beta]_{S}, S^{\prime}\right)$ the extremal length of the family of all closed curves on $S^{\prime}$ freely homotopic to $\beta$ in $S$. (For the definition of the extremal length, see [2].) We remark that if subdomains $S^{\prime}$ and $S^{\prime \prime}$ of $S$ satisfy $S^{\prime} \subset S^{\prime \prime}$ then

$$
\lambda\left([\beta]_{S}, S^{\prime \prime}\right) \leq \lambda\left([\beta]_{S}, S^{\prime}\right)
$$

follows. For a point $\tau=[R, \iota]$ in $T(S)$, let $K_{0}(\tau)$ be the dilatation of the unique extremal quasiconformal map from $S$ onto $R$ which induces the isomorphism $\iota: \pi_{1}(S) \rightarrow \pi_{1}(R)$. In other words, $\log K_{0}(\tau)$ is the Teichmüller distance between $\tau$ and the base point $[S$, id]. 
Lemma 2.6 ([5, p. 247]). Let $S$ be a Riemann surface of finite analytic type. Then there exists a positive constant $c$ and finitely many simple closed curves $\beta_{1}, \ldots, \beta_{N}$ on $S$ such that the inequality

$$
K_{0}(\tau) \leq c \max _{1 \leq l \leq N} \lambda\left(\iota\left[\beta_{l}\right]_{R}, R\right)
$$

holds for every point $\tau=[R, \iota]$ in $T(S)$.

Lemma 2.7 ([4, p. 218]). Let $S$ be a Riemann surface of finite analytic type and $\varphi \in A(S) \backslash\{0\}$. Then for every $\tau \in T(S)$

$$
K_{0}(\tau)^{-1}\|\varphi\|_{L^{1}(S)} \leq\left\|\tau_{*} \varphi\right\|_{L^{1}\left(S_{\tau}\right)} \leq K_{0}(\tau)\|\varphi\|_{L^{1}(S)} .
$$

Let $E$ be an allowable subset of a Riemann surface $S$ of finite type. Let $\mathfrak{F}(S, E)$ denote the set of pairs $\left(g, S_{g}\right)$, where $g: S \backslash E \rightarrow S_{g}$ is a type-preserving conformal embedding with respect to $S$. Set $\mathfrak{T}(S, E)=\left\{\left[S_{g}^{\mathrm{d}}, \iota_{g}\right] \in T\left(S^{\mathrm{d}}\right)\right.$ : $\left.\left(g, S_{g}\right) \in \mathfrak{F}(S, E)\right\}$.

Lemma 2.8. Let $E$ be an allowable subset of a Riemann surface $S$ of finite type and $\varphi \in A_{\mathrm{s}}(S) \backslash\{0\}$. Then, the set $\mathfrak{T}(S, E)$ is relatively compact in $T\left(S^{\mathrm{d}}\right)$ and there is a positive constant $c_{0}$ such that

$$
c_{0}^{-1}\|\varphi\|_{L^{1}(S)} \leq M_{h} \leq c_{0}\|\varphi\|_{L^{1}(S)}
$$

for every $\left(h, S_{h}\right) \in \mathfrak{F}(S, E)$.

Proof. For the double $S^{\mathrm{d}}$ we take a family of curves $\beta_{1}, \ldots, \beta_{N}$ and a positive constant $c$ as in Lemma 2.6. We may assume that each $\beta_{k}$ is contained in $S^{\mathrm{d}} \backslash E^{\mathrm{d}}$. For every $\left(h, S_{h}\right) \in \mathfrak{F}(S, E)$ set $\tau=\left[S_{h}^{\mathrm{d}}, \iota_{h}\right] \in T\left(S^{\mathrm{d}}\right)$. Lemma 2.7, together with $\left\|\varphi^{\mathrm{d}}\right\|_{L^{1}\left(S^{\mathrm{d}}\right)}=2\|\varphi\|_{L^{1}(S)}$ and $\left\|\varphi_{h}^{\mathrm{d}}\right\|_{L^{1}\left(S_{h}^{\mathrm{d}}\right)}=2\left\|\varphi_{h}\right\|_{L^{1}\left(S_{h}\right)}$, implies

$$
\frac{\|\varphi\|_{L^{1}(S)}}{K_{0}(\tau)} \leq\left\|\varphi_{h}\right\|_{L^{1}\left(S_{h}\right)} \leq K_{0}(\tau)\|\varphi\|_{L^{1}(S)} .
$$

Moreover $h^{\mathrm{d}}\left(S^{\mathrm{d}} \backslash E^{\mathrm{d}}\right) \subset S_{h}^{\mathrm{d}}$ and Lemma 2.6 imply

$$
\begin{aligned}
K_{0}(\tau) & \leq c \max _{1 \leq l \leq N} \lambda\left(\iota_{h}\left[\beta_{l}\right]_{S_{h}^{\mathrm{d}}}, S_{h}^{\mathrm{d}}\right) \\
& =c \max _{1 \leq l \leq N} \lambda\left(\left[h^{\mathrm{d}}\left(\beta_{l}\right)\right]_{S_{h}^{\mathrm{d}}}, S_{h}^{\mathrm{d}}\right) \\
& \leq c \max _{1 \leq l \leq N} \lambda\left(\left[h^{\mathrm{d}}\left(\beta_{l}\right)\right]_{S_{h}^{\mathrm{d}}}, h^{\mathrm{d}}\left(S^{\mathrm{d}} \backslash E^{\mathrm{d}}\right)\right) \\
& =c \max _{1 \leq l \leq N} \lambda\left(\left[\beta_{l}\right]_{S^{\mathrm{d}}}, S^{\mathrm{d}} \backslash E^{\mathrm{d}}\right)<\infty .
\end{aligned}
$$

The last constant is independent of $\left(h, S_{h}\right) \in \mathfrak{F}(S, E)$. This means that $\mathfrak{T}(S, E)$ is relatively compact in $T\left(S^{\mathrm{d}}\right)$. Moreover, letting

$$
c_{0}=c \max _{1 \leq l \leq N} \lambda\left(\left[\beta_{l}\right]_{S^{\mathrm{d}}}, S^{\mathrm{d}} \backslash E^{\mathrm{d}}\right),
$$


we have

$$
c_{0}^{-1}\|\varphi\|_{L^{1}(S)} \leq M_{h}=\left\|\varphi_{h}\right\|_{L^{1}\left(S_{h}\right)} \leq c_{0}\|\varphi\|_{L^{1}(S)}
$$

for every $\left(h, S_{h}\right) \in \mathfrak{F}(S, E)$.

\section{Proof of Theorem $\mathbf{1 . 3}$}

Take a sequence $\left\{\left(g_{n}, S_{g_{n}}\right)\right\}_{n} \subset \mathfrak{F}(S, E)$ such that

$$
\lim _{n \rightarrow \infty} M_{g_{n}}=\sup _{\left(h, S_{h}\right) \in \mathfrak{F}(S, E)} M_{h} \text {. }
$$

Set $\tau_{n}=\left[S_{g_{n}}^{\mathrm{d}}, \iota_{g_{n}}\right] \in T\left(S^{\mathrm{d}}\right)$. Let $\Gamma$ and $\Gamma_{n}(n \in \mathbb{N})$ be the normalized Fuchsian models $\left(\left[6\right.\right.$, p. 59]) of $S$ and $S_{g_{n}}$, respectively. Since $\left\{\left[S_{g_{n}}^{\mathrm{d}}, \iota_{g_{n}}\right]\right\}_{n}$ is relatively compact in $T\left(S^{\mathrm{d}}\right)$ by Lemma 2.8 , we may assume that the sequence $\left[S_{g_{n}}^{\mathrm{d}}, \iota_{g_{n}}\right]$ is convergent in $T\left(S^{\mathrm{d}}\right)$. Then the isomorphisms $\chi_{n}: \Gamma \rightarrow \Gamma_{n}$ induced by $\iota_{g_{n}}$ algebraically converge to an isomorphism $\chi_{\infty}: \Gamma \rightarrow \Gamma_{\infty}$. We denote by $S_{\infty}$ the quotient surface $\mathbb{D} / \Gamma_{\infty}$. Let $G$ be a Fuchsian model of $S \backslash E$ and $\rho: G \rightarrow \Gamma$ be a surjective homomorphism induced by the inclusion mapping $S \backslash E \hookrightarrow S$. For each $n \in \mathbb{N}$, set

$$
\rho_{n}=\chi_{n} \circ \rho: G \rightarrow \Gamma_{n} .
$$

Since $\chi_{n}$ converge to $\chi_{\infty}$, the homomorphisms $\rho_{n}$ converge to the surjective homomorphism

$$
\rho_{\infty}=\chi_{\infty} \circ \rho: G \rightarrow \Gamma_{\infty}
$$

Let $\tilde{g}_{n}: \mathbb{D} \rightarrow \mathbb{D}(n \in \mathbb{N})$ be a lift of $g_{n}: S \backslash E \rightarrow S_{g}$ associated with $\rho_{n}$, e.g.,

$$
\tilde{g}_{n} \circ A=\rho_{n}(A) \circ \tilde{g}_{n}
$$

for every $A \in G$. Since $\left\{\tilde{g}_{n}\right\}_{n}$ is normal, there is a subsequence which converges to a holomorphic map $\tilde{g}$ on $\mathbb{D}$ uniformly on any compact subset of $\mathbb{D}$. Then we obtain

$$
\tilde{g} \circ A=\rho_{\infty}(A) \circ \tilde{g}
$$

for every $A \in G$. If $\tilde{g}$ is a constant $c$, then $\Gamma_{\infty}$ has the common fixed point $c$, and thus $\Gamma_{\infty}$ is a cyclic group, which is not the case. So $\tilde{g}$ is non-constant and thus a holomorphic map into $\mathbb{D}$. Therefore $\tilde{g}$ descends to an injective holomorphic map $g$ of $S \backslash E$ into $S_{\infty}$ such that the homomorphism $(g)_{*}$ corresponds to $\rho_{\infty}$. We denote $S_{\infty}$ by $S_{g}$. Then it is easy to see that $\left(g, S_{g}\right) \in \mathfrak{F}(S, E)$.

Let $\hat{\Gamma}, \hat{\Gamma}_{n}(n \in \mathbb{N})$ and $\hat{\Gamma}_{\infty}$ be the normalized Fuchsian models of the compact Riemann surfaces $\hat{S}, \hat{S}_{g_{n}}$ and $\hat{S}_{g}$, which are the completion of the doubles $S^{\mathrm{d}}, S_{g_{n}}^{\mathrm{d}}$ and $S_{g}^{\mathrm{d}}$, respectively. (In the case when $\hat{S}$ is not hyperbolic, the proof will be simpler.) Let $f_{n}$ be a quasiconformal map of $S^{\mathrm{d}}$ onto $S_{g_{n}}^{\mathrm{d}}$ which represents $\left[S_{g_{n}}^{\mathrm{d}}, \iota_{g_{n}}\right] \in T\left(S^{\mathrm{d}}\right)$ and let $f$ be a quasiconformal map of $S^{\mathrm{d}}$ onto $S_{g}^{\mathrm{d}}$ representing the element $\left[S_{g}^{\mathrm{d}}, \iota_{g}\right] \in T\left(S^{\mathrm{d}}\right)$. Then $f_{n}$ and $f$ extend to quasiconformal mappings of $\hat{S}$ onto $\hat{S}_{g_{n}}$ and $\hat{S}_{g}$, respectively. Let $\hat{f}_{n}$ and $\hat{f}: \mathbb{D} \rightarrow \mathbb{D}$ be the normalized lifts of $f_{n}$ and $f$ to the universal covering space $\mathbb{D}$ over $\hat{S}_{g_{n}}$ and $\hat{S}_{g}$, respectively (see the following commutative diagram). 


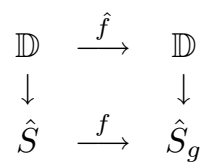

Let $\hat{\Omega}$ be a relatively compact Dirichlet fundamental domain of $\hat{\Gamma}$ with base point at the origin. Set $\hat{\Omega}_{n}=\hat{f}_{n}(\hat{\Omega})(n \in \mathbb{N})$ and $\hat{\Omega}_{\infty}=\hat{f}(\hat{\Omega})$. Then $\hat{\Omega}_{n}$ and $\hat{\Omega}_{\infty}$ are fundamental domains of $\hat{\Gamma}_{n}$ and $\hat{\Gamma}_{\infty}$, respectively. Let $\hat{\varphi}_{g_{n}}$ be a lift of $\varphi_{g_{n}}^{\mathrm{d}}$ to the universal covering space $\mathbb{D}$ over $\hat{S}_{g_{n}}$. For each $n \in \mathbb{N}, \hat{\varphi}_{g_{n}}$ is a meromorphic quadratic differential on $\mathbb{D}$ with at most simple poles. Let $D$ be the subdomain of $\mathbb{D}$ which is obtained from $\mathbb{D}$ by removing the points corresponding to the punctures of $S_{g}^{\mathrm{d}}$.

Lemma 3.1. The family $\left\{\hat{\varphi}_{g_{n}}\right\}_{n}$ is normal on $D$.

Proof. It is sufficient to show that $\left\{\left\|\hat{\varphi}_{g_{n}}\right\|_{L^{1}(K)}\right\}_{n}$ is bounded for any compact subset $K$ of $\mathbb{D}$. Let

$$
\left\{A \in \hat{\Gamma}_{\infty}: A\left(\hat{\Omega}_{\infty} \cup \partial \hat{\Omega}_{\infty}\right) \cap K \neq \emptyset\right\}=\left\{A_{1}, \ldots, A_{m_{0}}\right\} .
$$

Then, the euclidean distance $d_{\infty}$ between $K$ and $\left(\cup_{i=1}^{m_{0}} A_{i}\left(\hat{\Omega}_{\infty}\right)\right)^{c}$ is positive. Since we can assume that $\hat{f}_{n}$ converge to $\hat{f}$ uniformly on any compact subset of $\mathbb{D}$, for each $A_{i}\left(1 \leq i \leq m_{0}\right)$ the Möbius transformations

$$
A_{i, n}=\left(\hat{f}_{n} \circ \hat{f}^{-1}\right) \circ A_{i} \circ\left(\hat{f}_{n} \circ \hat{f}^{-1}\right)^{-1} \in \hat{\Gamma}_{n}
$$

converge to $A_{i}$ as $n \rightarrow \infty$. Then the distances $d_{n}$ between $K$ and $\left(\cup_{i=1}^{m_{0}} A_{i, n}\left(\hat{\Omega}_{n}\right)\right)^{c}$ converge to $d_{\infty}$. Hence $d_{n}>0$ for sufficiently large $n$. This implies

$$
K \subset \bigcup_{i=1}^{m_{0}} A_{i, n}\left(\hat{\Omega}_{n} \cup \partial \hat{\Omega}_{n}\right)
$$

Then

$$
\#\left\{A \in \hat{\Gamma}_{n}: A\left(\hat{\Omega}_{n} \cup \partial \hat{\Omega}_{n}\right) \cap K \neq \emptyset\right\} \leq m_{0}
$$

for sufficiently large $n$. This result together with boundedness of $\left\{M_{g_{n}}\right\}_{n}$ (Lemma 2.8) implies that $\left\{\left\|\hat{\varphi}_{g_{n}}\right\|_{L^{1}(K)}\right\}_{n}$ is bounded.

Lemma 3.2. $M_{g}=\sup \left\{M_{h}:\left(h, S_{h}\right) \in \mathfrak{F}(S, E)\right\}$.

Proof. By Lemma 3.1, there is a subsequence of $\left\{\hat{\varphi}_{g_{n}}\right\}_{n}$ which converges uniformly on any compact subset of $D$ to a holomorphic function $\hat{\varphi}_{\infty}$. For each $n \in \mathbb{N}, \hat{\varphi}_{g_{n}}$ is $\hat{\Gamma}_{n}$-invariant, that is,

$$
\left(\hat{\varphi}_{g_{n}} \circ A_{n}\right)\left(A_{n}^{\prime}\right)^{2}=\hat{\varphi}_{g_{n}}
$$


for every $A_{n} \in \hat{\Gamma}_{n}$. Then we see that $\hat{\varphi}_{\infty}$ is $\hat{\Gamma}_{\infty}$-invariant, so we can project $\hat{\varphi}_{\infty}$ to a holomorphic quadratic differential $\varphi_{\infty}^{*}$ on $S_{g}^{\mathrm{d}}$. Moreover for any compact subset $K$ of $D$,

$$
\left\|\hat{\varphi}_{\infty}\right\|_{L^{1}(K)}=\lim _{n \rightarrow \infty}\left\|\hat{\varphi}_{g_{n}}\right\|_{L^{1}(K)} \leq 2 c_{0}\|\varphi\|_{L^{1}(S)}
$$

holds, where $c_{0}$ is the positive number obtained in Lemma 2.8. Hence we can see that $\hat{\varphi}_{\infty}$ is integrable on $\hat{\Omega}_{\infty}$.

Next we show that the integrable holomorphic quadratic differential $\varphi_{\infty}^{*}$ on $S_{g}^{\mathrm{d}}$ is symmetric relative to $\partial S_{g}$. Let $\Lambda_{n}(n \in \mathbb{N})$ and $\Lambda_{\infty}$ be the limit sets of $\Gamma_{n}$ and $\Gamma_{\infty}$, respectively. Let $\tilde{\varphi}_{g_{n}}(n \in \mathbb{N})$ be the lift of $\varphi_{g_{n}}^{\mathrm{d}}$ to the covering space $\overline{\mathbb{C}} \backslash \Lambda_{n}$ over $S_{g_{n}}^{\mathrm{d}}$ and let $\tilde{\varphi}_{\infty}$ be the lift of $\varphi_{\infty}^{*}$ to $\overline{\mathbb{C}} \backslash \Lambda_{\infty}$. By assumption, for each $n \in \mathbb{N}, \tilde{\varphi}_{g_{n}}$ satisfies

$$
\overline{\tilde{\varphi}_{g_{n}}(1 / \bar{z})} \cdot z^{-4}=\tilde{\varphi}_{g_{n}}(z) \text { on } \overline{\mathbb{C}} \backslash \Lambda_{n} .
$$

Since $\tilde{\varphi}_{g_{n}}$ converge to $\tilde{\varphi}_{\infty}$ uniformly on any compact subset of $\overline{\mathbb{C}} \backslash \Lambda_{\infty}$, we have

$$
\overline{\tilde{\varphi}_{\infty}(1 / \bar{z})} \cdot z^{-4}=\tilde{\varphi}_{\infty}(z) \text { on } \overline{\mathbb{C}} \backslash \Lambda_{\infty} .
$$

Hence $\tilde{\varphi}_{\infty}$ is symmetric relative to $\partial \mathbb{D}$, that is, the projection $\varphi_{\infty}^{*}$ of $\tilde{\varphi}_{\infty}$ to $S_{g}^{\mathrm{d}}$ is symmetric relative to $\partial S_{g}$. We denote by $\varphi_{\infty}$ the restriction of $\varphi_{\infty}^{*}$ to $S_{g}$.

Now we show

$$
\lim _{n \rightarrow \infty}\left\|\varphi_{g_{n}}\right\|_{L^{1}\left(S_{g_{n}}\right)}=\left\|\varphi_{\infty}\right\|_{L^{1}\left(S_{g}\right)} .
$$

Let $p_{1}, \ldots, p_{k}$ be all the points in $\hat{\Omega}_{\infty} \cup \partial \hat{\Omega}_{\infty}$ corresponding to the punctures of $S_{g}^{\mathrm{d}}$. We may assume that all $p_{1}, \ldots, p_{k}$ are points of $\hat{\Omega}_{\infty}$. Set

$$
p_{i, n}=\hat{f}_{n} \circ \hat{f}^{-1}\left(p_{i}\right) \quad(n \in \mathbb{N}, 1 \leq i \leq k) .
$$

Then $p_{i, n} \rightarrow p_{i}$ as $n \rightarrow \infty$.

For sufficiently small $\varepsilon>0$, let $V_{i} \subset \hat{\Omega}_{\infty}(1 \leq i \leq k)$ be the open disk of radius $\varepsilon$ centered at $p_{i}$ such that $V_{i} \cap V_{j}=\phi$ if $i \neq j$. Since holomorphic functions $\left(z-p_{i, n}\right) \hat{\varphi}_{g_{n}}(z)(n \in \mathbb{N})$ on $V_{i}$ converge to $\left(z-p_{i}\right) \hat{\varphi}_{\infty}(z)$ uniformly on $V_{i}$, the residues $c_{i, n}=\operatorname{Res}\left(\hat{\varphi}_{g_{n}}, p_{i, n}\right)$ converge to $c_{i}=\operatorname{Res}\left(\hat{\varphi}_{\infty}, p_{i}\right)$ as $n \rightarrow \infty$. Moreover we can also see that holomorphic functions

$$
\hat{\varphi}_{g_{n}}(z)-\frac{c_{i, n}}{z-p_{i, n}}
$$

converge to

$$
\hat{\varphi}_{\infty}(z)-\frac{c_{i}}{z-p_{i}}
$$

uniformly on $V_{i}$. We take the union $\bigcup_{n \geq n_{0}}\left(\hat{\Omega}_{n} \cup \partial \hat{\Omega}_{n}\right)$ for sufficiently large $n_{0} \in \mathbb{N}$. Then $\hat{\varphi}_{g_{n}}$ converge to $\hat{\varphi}_{\infty}$ uniformly on the compact set

$$
\left(\bigcup_{n \geq n_{0}}\left(\hat{\Omega}_{n} \cup \partial \hat{\Omega}_{n}\right) \bigcup\left(\hat{\Omega}_{\infty} \cup \partial \hat{\Omega}_{\infty}\right)\right) \backslash \bigcup_{i=1}^{k} V_{i} .
$$


Set

$$
C=\max _{1 \leq i \leq k}\left|c_{i}\right|
$$

Then we have

$$
\begin{aligned}
& \limsup _{n \rightarrow \infty}\left|\left\|\hat{\varphi}_{g_{n}}\right\|_{L^{1}\left(\hat{\Omega}_{n}\right)}-\left\|\hat{\varphi}_{\infty}\right\|_{L^{1}\left(\hat{\Omega}_{\infty}\right)}\right| \\
& \leq \limsup \sum_{i=1}^{k}\left(\left\|\frac{c_{i}}{z-p_{i}}\right\|_{L^{1}\left(V_{i}\right)}+\left\|\frac{c_{i, n}}{z-p_{i, n}}\right\|_{L^{1}\left(V_{i}\right)}\right) \\
& \quad+\limsup \sum_{i=1}^{k}\left\|\left(\hat{\varphi}_{g_{n}}-\frac{c_{i, n}}{z-p_{i, n}}\right)-\left(\hat{\varphi}_{\infty}-\frac{c_{i}}{z-p_{i}}\right)\right\|_{L^{1}\left(V_{i}\right)} \\
& \quad+\limsup \left|\left\|\hat{\varphi}_{g_{n}}\right\|_{L^{1}\left(\hat{\Omega}_{n} \backslash \cup_{i=1}^{k} V_{i}\right)}-\left\|\hat{\varphi}_{\infty}\right\|_{L^{1}\left(\hat{\Omega}_{\infty} \backslash \cup_{i=1}^{k} V_{i}\right)}\right| \\
& \leq C k 4 \pi \varepsilon .
\end{aligned}
$$

Thus

$$
\lim _{n \rightarrow \infty}\left\|\varphi_{g_{n}}\right\|_{L^{1}\left(S_{g_{n}}\right)}=\left\|\varphi_{\infty}\right\|_{L^{1}\left(S_{g}\right)},
$$

and we obtain $\left\|\varphi_{\infty}\right\|_{L^{1}\left(S_{g}\right)}=\sup _{\left(h, S_{h}\right) \in \mathfrak{F}(S, E)} M_{h}$. Moreover, by Lemma 2.8, we have

$$
\left\|\varphi_{\infty}\right\|_{L^{1}\left(S_{g}\right)}=\lim _{n \rightarrow \infty}\left\|\varphi_{g_{n}}\right\|_{L^{1}\left(S_{g_{n}}\right)} \geq c_{0}^{-1}\|\varphi\|_{L^{1}(S)} .
$$

Therefore $\varphi_{\infty} \in A_{\mathrm{s}}\left(S_{g}\right) \backslash\{0\}$.

Next we show $\varphi_{\infty}=\varphi_{g}$ on $S_{g}$. Take any curve $\gamma \in \mathfrak{S}\left(S_{g}^{\mathrm{d}}\right)$ and sufficiently small $\varepsilon>0$. Set $\gamma_{n}=f_{n} \circ f^{-1}(\gamma)(n \in \mathbb{N})$. Assume that $A_{\infty} \in \hat{\Gamma}_{\infty}$ corresponds to the homotopy class $[\gamma]$ on $\hat{S}_{g}$. Then

$$
A_{n}=\left(\hat{f}_{n} \circ \hat{f}^{-1}\right) \circ A_{\infty} \circ\left(\hat{f}_{n} \circ f^{-1}\right)^{-1}
$$

corresponds to the homotopy class $\left[\gamma_{n}\right]$ on $\hat{S}_{g_{n}}$.

Let $\alpha_{n} \in \mathfrak{S}\left(S_{g_{n}}^{\mathrm{d}}\right)$ be a $\varphi_{g_{n}}^{\mathrm{d}}$-geodesic curve freely homotopic to $\gamma_{n}$ on $S_{g_{n}}^{\mathrm{d}}$, that is, $\alpha_{n}$ satisfies $\left[\alpha_{n}\right]=\left[\gamma_{n}\right]$ on $S_{g_{n}}^{\mathrm{d}}$ and $\ell_{\varphi_{g_{n}}^{\mathrm{d}}}\left[\gamma_{n}\right]=\ell_{\varphi_{g_{n}}^{\mathrm{d}}}\left(\alpha_{n}\right)$, where $\ell_{\varphi_{g_{n}}^{\mathrm{d}}}\left(\alpha_{n}\right)$ is the length

$$
\int_{\alpha_{n}}\left|\sqrt{\varphi_{g_{n}}^{\mathrm{d}}(z)} d z\right|
$$

of $\alpha_{n}$ associated with $\varphi_{g_{n}}^{\mathrm{d}}$ and $\ell_{\varphi_{g_{n}}^{\mathrm{d}}}\left[\gamma_{n}\right]$ is that of homotopy class $\left[\gamma_{n}\right]$ defined by

$$
\inf _{\beta} \ell_{\varphi_{g_{n}}^{\mathrm{d}}}(\beta),
$$

where the infimum is taken over all closed curves $\beta$ freely homotopic to $\gamma_{n}$ on $S_{g_{n}}^{\mathrm{d}}$. 
We remark that the geodesic $\alpha_{n}$ may pass through punctures of $S_{g_{n}}^{\mathrm{d}}$. Therefore, we should interpret the condition $\left[\alpha_{n}\right]=\left[\gamma_{n}\right]$ on $S_{g_{n}}^{\mathrm{d}}$ in an appropriate way.

It is known [11, Theorem 24.1] that each $\alpha_{n}$ satisfies height $\varphi_{\varphi_{n}}\left[\gamma_{n}\right]=$ height $_{\varphi_{g_{n}}^{\mathrm{d}}}\left(\alpha_{n}\right)$. Let $\hat{\alpha}_{n}(n \in \mathbb{N})$ be a lift of $\alpha_{n}$ to the universal covering space $\mathbb{D}$ over $\hat{S}_{g_{n}}$ such that $\hat{\alpha}_{n}$ is a closed arc starting from a point on $\partial \hat{\Omega}_{n}$. We parametrize each $\hat{\alpha}_{n}$ by the $\hat{\varphi}_{g_{n}}$-length $s$ and we set $l(n)=\ell_{\hat{\varphi}_{g_{n}}}\left(\hat{\alpha}_{n}\right)$. Then $\{l(n)\}_{n}$ is bounded because the set $\left\{\left[S_{g_{n}}^{\mathrm{d}}, \iota_{g_{n}}\right]\right\}_{n}$ is relatively compact in $T\left(S^{\mathrm{d}}\right)$ by Lemma 2.8. Thus we may assume that $l(n)$ converge to a positive constant $l_{\infty}$. For each $n \in \mathbb{N}$ by re-parametrizing $\hat{\alpha}_{n}$ by $\hat{\alpha}_{n}(l(n) s)$, we may further assume that for every $n \in \mathbb{N}, \hat{\alpha}_{n}$ is defined on $[0,1]$. The curve $\alpha_{n}(n \in \mathbb{N})$ is analytic except for finitely many points where $\varphi_{g_{n}}^{\mathrm{d}}$ vanishes. Since the number of punctures of $S_{g_{n}}^{\mathrm{d}}$ are the same for every $n \in \mathbb{N}$, the number of zeros of $\varphi_{g_{n}}^{\mathrm{d}}$ is uniformly bounded for $n \in \mathbb{N}$. And $\{l(n)\}_{n}$ is also bounded. Hence the number of singularities of $\hat{\alpha}_{n}$ are uniformly bounded. Then we can assume that there exists an integer $N_{0}$, and real numbers $t_{1}, t_{2}, \ldots, t_{N_{0}} \in[0,1]\left(t_{1} \leq t_{2} \leq \cdots \leq\right.$ $t_{N_{0}}$ ) such that the number of critical points of $\hat{\alpha}_{n}$ is $N_{0}$ for all $n \in \mathbb{N}$ and for each $i=1, \ldots, N_{0}$, the $i$-th critical point $t_{n, i}$ of $\hat{\alpha}_{n}$ converge to $t_{i}$ as $n \rightarrow \infty$. We can easily see that the family $\left\{\hat{\alpha}_{n}\right\}_{n \in \mathbb{N}}$ is equicontinuous. Then, together with uniform boundedness of $\left\{\hat{\alpha}_{n}\right\}_{n \in \mathbb{N}}$, we may assume that $\hat{\alpha}_{n}$ uniformly converge to a continuous function $\hat{\alpha}_{\infty}$ on $[0,1]$. The image $\hat{\alpha}_{\infty}([0,1])$ is a lift of a closed curve on $\hat{S}_{g}$.

Now we can show that height $\hat{\varphi}_{g_{n}}\left(\hat{\alpha}_{n}\right)$ converge to height $\hat{\varphi}_{\infty}\left(\hat{\alpha}_{\infty}\right)$. Let $s_{0}$ be any point on $(0,1) \backslash\left\{t_{1}, \ldots, t_{N_{0}}\right\}$ such that $\hat{\varphi}_{\infty}\left(\hat{\alpha}_{\infty}\left(s_{0}\right)\right) \neq 0$ and $\hat{\alpha}_{\infty}\left(s_{0}\right) \in$ $D$. We can take a neighborhood $U$ of $s_{0}$ in $(0,1)$ such that for every $n \in$ $\mathbb{N}, \hat{\varphi}_{g_{n}}\left(\hat{\alpha}_{n}(s)\right) \neq 0$ on $U \cup \partial U$ and $\hat{\alpha}_{n}(U \cup \partial U)$ does not contain any points corresponding to punctures of $S_{g_{n}}^{\mathrm{d}}$. Let $\hat{\zeta}_{n}(n \in \mathbb{N})$ and $\hat{\zeta}_{\infty}$ be the natural parameters near $\hat{\alpha}_{n}\left(s_{0}\right)$ and $\hat{\alpha}_{\infty}\left(s_{0}\right)$, which are represented by

$$
\hat{\zeta}_{n}=\int_{\hat{\alpha}_{n}\left(s_{0}\right)}^{z} \sqrt{\hat{\varphi}_{g_{n}}(z)} d z
$$

and

$$
\hat{\zeta}_{\infty}=\int_{\hat{\alpha}_{\infty}\left(s_{0}\right)}^{z} \sqrt{\hat{\varphi}_{\infty}(z)} d z,
$$

respectively. For every $n \in \mathbb{N}, \alpha_{n}$ is geodesic relative to $\varphi_{g_{n}}^{\mathrm{d}}$-length, so we see that

$$
\frac{d \hat{\zeta}_{n}}{\left|d \hat{\zeta}_{n}\right|}=e^{i \theta_{n}} \text { on } U
$$

for a constant $\theta_{n} \in[0,2 \pi)$. For any subsequence $\left\{\theta_{n_{k}}\right\}_{k}$ which is converging to 
some $\theta_{\infty} \in[0,2 \pi]$

$$
\begin{aligned}
\frac{d \hat{\alpha}_{n_{k}}}{d s} & =\frac{l\left(n_{k}\right) d \hat{\alpha}_{n_{k}}}{\left|d \hat{\zeta}_{n_{k}}\right|} \\
& =\frac{l\left(n_{k}\right) d \hat{\alpha}_{n_{k}}}{d \hat{\zeta}_{n_{k}}} \cdot \frac{d \hat{\zeta}_{n_{k}}}{\left|d \hat{\zeta}_{n_{k}}\right|} \\
& =\frac{l\left(n_{k}\right)}{\sqrt{\hat{\varphi}_{g_{n_{k}}}\left(\hat{\alpha}_{n_{k}}\right)}} \cdot e^{i \theta_{n_{k}}} \\
& \stackrel{k \rightarrow \infty}{\longrightarrow} \frac{l_{\infty}}{\sqrt{\hat{\varphi}_{\infty}\left(\hat{\alpha}_{\infty}\right)}} \cdot e^{i \theta_{\infty}} .
\end{aligned}
$$

The convergence is uniform on $U$, so we can see that $\hat{\alpha}_{\infty}$ is of the class $C^{1}$ on $U$ and

$$
\frac{d \hat{\alpha}_{\infty}}{d s}=\lim _{k \rightarrow \infty} \frac{d \hat{\alpha}_{n_{k}}}{d s}=\frac{l_{\infty}}{\sqrt{\hat{\varphi}_{\infty}\left(\hat{\alpha}_{\infty}\right)}} \cdot e^{i \theta_{\infty}} .
$$

Since the argument $\theta_{\infty}$ is independent of the choice of subsequence, the sequence $\left\{\theta_{n}\right\}_{n}$ itself is converging and we obtain

$$
\frac{d \hat{\alpha}_{\infty}}{d s}=\lim _{n \rightarrow \infty} \frac{d \hat{\alpha}_{n}}{d s} \text { on } U .
$$

Consequently the curve $\hat{\alpha}_{\infty}$ satisfies

$$
\frac{d \hat{\alpha}_{\infty}}{d s}=\lim _{n \rightarrow \infty} \frac{d \hat{\alpha}_{n}}{d s} \text { on }(0,1)
$$

except for finitely many points. Then Lebesgue's convergence theorem yields

$$
\lim _{n \rightarrow \infty} \text { height }_{\hat{\varphi}_{g_{n}}}\left(\hat{\alpha}_{n}\right)=\text { height }_{\hat{\varphi}_{\infty}}\left(\hat{\alpha}_{\infty}\right)
$$

because

$$
\left|\operatorname{Im}\left(\sqrt{\hat{\varphi}_{g_{n}}\left(\hat{\alpha}_{n}\right)} \frac{d \hat{\alpha}_{n}}{d s}\right)\right| \leq\left|\sqrt{\hat{\varphi}_{g_{n}}\left(\hat{\alpha}_{n}\right)} \frac{d \hat{\alpha}_{n}}{d s}\right|=l(n) \leq \sup _{n \in \mathbb{N}} l(n)<\infty
$$

for every $n \in \mathbb{N}$ and

$$
\lim _{n \rightarrow \infty}\left|\operatorname{Im}\left(\sqrt{\hat{\varphi}_{g_{n}}\left(\hat{\alpha}_{n}(s)\right)} \frac{d \hat{\alpha}_{n}}{d s}\right)\right|=\left|\operatorname{Im}\left(\sqrt{\hat{\varphi}_{\infty}\left(\hat{\alpha}_{\infty}(s)\right)} \frac{d \hat{\alpha}_{\infty}}{d s}\right)\right| \text { a.e. on }[0,1] \text {. }
$$

If there exists $s_{1} \in[0,1]$ such that $\hat{\alpha}_{\infty}\left(s_{1}\right)$ corresponds to a puncture of $S_{g}^{\mathrm{d}}$, we modify the curve $\hat{\alpha}_{\infty}$ locally in a neighborhood of $s_{1}$ to a curve $\hat{\alpha}_{\infty}^{\prime}$ so that the projection on $S_{g}^{\mathrm{d}}$ is homotopic to $\gamma$ on $S_{g}^{\mathrm{d}}$. It is possible to choose $\hat{\alpha}_{\infty}^{\prime}$ so that $\ell_{\hat{\varphi}_{\infty}}\left(\hat{\alpha}_{\infty}^{\prime}\right)$ is as close to $\ell_{\hat{\varphi}_{\infty}}\left(\hat{\alpha}_{\infty}\right)$ as we want. Therefore, for any $\varepsilon>0$, 
there is a curve $\hat{\alpha}_{\infty}^{\prime}$ so that

$$
\begin{aligned}
\operatorname{height}_{\varphi_{\infty}^{\mathrm{d}}}[\gamma] & \leq \operatorname{height}_{\hat{\varphi}_{\infty}}\left(\hat{\alpha}_{\infty}^{\prime}\right) \\
& <\operatorname{height}_{\hat{\varphi}_{\infty}}\left(\hat{\alpha}_{\infty}\right)+\varepsilon \\
& =\lim _{n \rightarrow \infty} \operatorname{height}_{\hat{\varphi}_{g_{n}}}\left(\hat{\alpha}_{n}\right)+\varepsilon \\
& =\lim _{n \rightarrow \infty} \operatorname{height}_{\varphi_{g_{n}}^{\mathrm{d}}}\left[\gamma_{n}\right]+\varepsilon \\
& =\operatorname{height}_{\varphi_{g}^{\mathrm{d}}}[\gamma]+\varepsilon .
\end{aligned}
$$

Then we have the inequality height ${ }_{\varphi_{\infty}^{\mathrm{d}}}[\gamma] \leq$ height $_{\varphi_{g}^{\mathrm{d}}}[\gamma]$. In particular, by Proposition 2.4,

$$
\left\|\varphi_{\infty}^{\mathrm{d}}\right\|_{L^{1}\left(S_{g}^{\mathrm{d}}\right)} \leq\left\|\varphi_{g}^{\mathrm{d}}\right\|_{L^{1}\left(S_{g}^{\mathrm{d}}\right)} .
$$

On the other hand, from maximality of $\left\|\varphi_{\infty}\right\|_{L^{1}\left(S_{g}\right)}$ in $\left\{M_{h} ;\left(h, S_{h}\right) \in \mathfrak{F}(S, E)\right\}$ we have

$$
\left\|\varphi_{g}^{\mathrm{d}}\right\|_{L^{1}\left(S_{g}^{\mathrm{d}}\right)} \leq\left\|\varphi_{\infty}^{\mathrm{d}}\right\|_{L^{1}\left(S_{g}^{\mathrm{d}}\right)} .
$$

Therefore $\left\|\varphi_{\infty}^{\mathrm{d}}\right\|_{L^{1}\left(S_{g}^{\mathrm{d}}\right)}=\left\|\varphi_{g}^{\mathrm{d}}\right\|_{L^{1}\left(S_{g}^{\mathrm{d}}\right)}$ holds, and by Proposition 2.4, we have

$$
\varphi_{\infty}^{\mathrm{d}}=\varphi_{g}^{\mathrm{d}} \text { on } S_{g}^{\mathrm{d}} .
$$

Lemma 3.3. $\quad E_{g}=S_{g} \backslash g(S \backslash E)$ has measure zero.

Proof. If $E_{g}$ has positive measure, we can find a Beltrami differential $\nu$ on $S_{g}^{\mathrm{d}}$ so that $\nu=0$ on $S_{g}^{\mathrm{d}} \backslash E_{g}^{\mathrm{d}}$ and $\iint_{S_{g}^{\mathrm{d}}} \nu \varphi_{g}^{\mathrm{d}}>0$.

For sufficiently small $t>0$, let $f^{t \nu}$ be a quasiconformal map of $S_{g}^{\mathrm{d}}$ onto the double $S_{t \nu}^{\mathrm{d}}$ of a Riemann surface $S_{t \nu}$ with Beltrami coefficient $t \nu$. Let $\tau(t)=\left[S_{t \nu}^{\mathrm{d}},\left(f^{t \nu}\right)_{*}\right] \in T\left(S_{g}^{\mathrm{d}}\right)$. For $\varphi_{g} \in A_{\mathrm{s}}\left(S_{g}\right) \backslash\{0\}$ we set $\varphi_{t \nu}=\left.\tau(t)_{*} \varphi_{g}^{\mathrm{d}}\right|_{S_{t \nu}} \in$ $A_{\mathrm{s}}\left(S_{t \nu}\right) \backslash\{0\}$ whose heights on $S_{t \nu}^{\mathrm{d}}$ are equal to those of $\varphi_{g}^{\mathrm{d}}$ on $S_{g}^{\mathrm{d}}$. By the variational formula [4, p. 217], we have

$\log \left\|\varphi_{t \nu}^{\mathrm{d}}\right\|_{L^{1}\left(S_{t \nu}^{\mathrm{d}}\right)}=\log \left\|\varphi_{g}^{\mathrm{d}}\right\|_{L^{1}\left(S_{g}^{\mathrm{d}}\right)}+\frac{2}{\left\|\varphi_{g}^{\mathrm{d}}\right\|_{L^{1}\left(S_{g}^{\mathrm{d}}\right)}} \operatorname{Re} \iint_{S_{g}^{\mathrm{d}}} t \nu \varphi_{g}^{\mathrm{d}} d x d y+o\left(\|t \nu\|_{\infty}\right)$.

Then we can see

$$
\left\|\varphi_{t \nu}\right\|_{L^{1}\left(S_{t \nu}\right)}>\left\|\varphi_{g}\right\|_{L^{1}\left(S_{g}\right)}
$$

for sufficiently small $t>0$. On the other hand, $\left(f^{t \nu} \circ g, S_{t \nu}\right) \in \mathfrak{F}(S, E)$ for small $t>0$, and the maximality of $\left\|\varphi_{g}\right\|_{L^{1}\left(S_{g}\right)}$ yields the inequality

$$
\left\|\varphi_{t \nu}\right\|_{L^{1}\left(S_{t \nu}\right)} \leq\left\|\varphi_{g}\right\|_{L^{1}\left(S_{g}\right)}
$$

which is a contradiction. 
Lemma 3.4. A component of $E_{g}$ is either

(i) a horizontal arc of $\varphi_{g}$ or,

(ii) a connected union of finitely many horizontal arcs and critical points of $\varphi_{g}$.

In particular, $E_{g}$ is an allowable horizontal slit with respect to $\varphi_{g}$.

Proof. Fix any component $J$ of $E_{g}$. We consider the obstacle problem for $\left(S_{g}, J, \varphi_{g}\right)$. Then there is a solution $\left(h, S_{h}\right) \in \mathfrak{F}\left(S_{g}, J\right)$ and $\varphi_{h} \in A_{\mathrm{s}}\left(S_{h}\right) \backslash\{0\}$ satisfying

$$
\left\|\varphi_{h}\right\|_{L^{1}\left(S_{h}\right)}=\sup \left\{\left\|\varphi_{f}\right\|_{L^{1}\left(S_{f}\right)}:\left(f, S_{f}\right) \in \mathfrak{F}\left(S_{g}, J\right)\right\} .
$$

From the conclusion of Fehlmann and Gardiner [3, Theorem 1] we know that $S_{g} \backslash h\left(S_{g} \backslash J\right)$ is a possibly branched arc on horizontal trajectories of $\varphi_{h}$. Since $\left(h \circ g, S_{h}\right) \in \mathfrak{F}(S, E)$, we have $\left\|\varphi_{h}\right\|_{L^{1}\left(S_{h}\right)} \leq\left\|\varphi_{g}\right\|_{L^{1}\left(S_{g}\right)}$. On the other hand, (id, $\left.S_{g}\right) \in \mathfrak{F}\left(S_{g}, J\right)$ yields the inequality $\left\|\varphi_{g}\right\|_{L^{1}\left(S_{g}\right)} \leq\left\|\varphi_{h}\right\|_{L^{1}\left(S_{h}\right)}$. Thus (id, $\left.S_{g}\right) \in \mathfrak{F}\left(S_{g}, J\right)$ attains the maximum. By Theorem 2 in [3], we conclude that the component $J$ satisfies either (i) or (ii) in the lemma.

We have proved Theorem 1.3 completely.

\section{Proof of Theorem 1.4}

Let $E_{n} \subset S(n \in \mathbb{N})$ be an allowable subset of $S$ such that each $E_{n}$ is a disjoint union of finitely many closed analytic disks and satisfying $E_{n} \supset$ $E_{n+1} \supset \cdots$ and $\cap_{n=1}^{\infty} E_{n}=E$. Set $U_{n}=S \backslash E_{n}(n \in \mathbb{N})$. (Consider a regular exhaustion $\left\{R_{n}\right\}_{n \in \mathbb{N}}$ of the Riemann surface $\hat{S} \backslash E^{\text {d }}$ and set $U_{n}=S \cap R_{n}$ and $E_{n}=S \backslash U_{n}$ for sufficiently large $n$ 's; cf. [1, p. 144].) First we show that we can obtain a solution $\left(h_{\infty}, S_{h_{\infty}}\right) \in \mathfrak{F}(S, E)$ of the obstacle problem for $(S, E, \varphi)$ from the family of solutions $\left(h_{n}, S_{h_{n}}\right) \in \mathfrak{F}\left(S, E_{n}\right)$ for $\left(S, E_{n}, \varphi\right)(n \in \mathbb{N})$.

For each $n \in \mathbb{N}$, by considering the obstacle problem for $\left(S, E_{n}, \varphi\right)$, we obtain a solution $\left(h_{n}, S_{h_{n}}\right) \in \mathfrak{F}\left(S, E_{n}\right)$. Let $\tau_{n}=\left[S_{h_{n}}^{\mathrm{d}}, \iota_{h_{n}}\right] \in T\left(S^{\mathrm{d}}\right)$. We can see in a similar manner to Section 2 that the set $\left\{\tau_{n}\right\}_{n} \subset T\left(S^{\mathrm{d}}\right)$ is relatively compact in $T\left(S^{\mathrm{d}}\right)$. In fact, we may assume that the curve family $\beta_{1}, \ldots, \beta_{N}$ in Lemma 2.6 is contained in $S^{\mathrm{d}} \backslash U_{1}^{\mathrm{d}}$. Then the dilatation $K_{0}\left(\tau_{n}\right)$ of the extremal quasiconformal map of the Teichmüller class $\tau_{n}$ satisfies

$$
K_{0}\left(\tau_{n}\right) \leq c \max _{1 \leq l \leq N} \lambda\left(\left[\beta_{l}\right]_{U_{1}^{\mathrm{d}}}, U_{1}^{\mathrm{d}}\right)=: c_{1}
$$

for every $n \in \mathbb{N}$. Since the constant $c_{1}$ is independent of $n \in \mathbb{N},\left\{\tau_{n}\right\}_{n}$ is relatively compact in $T\left(S^{\mathrm{d}}\right)$. We can also see

$$
c_{1}^{-1}\|\varphi\|_{L^{1}(S)} \leq\left\|\varphi_{h_{n}}\right\|_{L^{1}\left(S_{h_{n}}\right)} \leq c_{1}\|\varphi\|_{L^{1}(S)}
$$

for every $n \in \mathbb{N}$. By the same argument as in the proof of Theorem 1.3, we obtain a subsequence $\left\{\tau_{n_{k}}\right\}_{k}$ and a point $\tau_{\infty}=\left[S_{h_{\infty}}, \iota_{h_{\infty}}\right] \in T\left(S^{\mathrm{d}}\right)$ associated with the element $\left(h_{\infty}, S_{h_{\infty}}\right) \in \mathfrak{F}(S, E)$ such that $\tau_{n_{k}}$ converge to $\tau_{\infty}$ in $T\left(S^{\mathrm{d}}\right)$, 
and the lift of $h_{n_{k}}^{\mathrm{d}}$ to the covering space $\mathbb{D}$ over $S^{\mathrm{d}} \backslash E^{\mathrm{d}}$ converge to that of $h_{\infty}^{\mathrm{d}}$ uniformly on any compact subset of $\mathbb{D}$. For brevity, we renumber $n_{k}$ by $k$. For every $\left(h, S_{h}\right) \in \mathfrak{F}(S, E)$ and every $n \in \mathbb{N}$, by considering the restriction of $h$ to $U_{n}$, we have

$$
\left\|\varphi_{h}\right\|_{L^{1}\left(S_{h}\right)} \leq\left\|\varphi_{h_{n}}\right\|_{L^{1}\left(S_{h_{n}}\right)}
$$

Since $\left\|\varphi_{h_{n}}\right\|_{L^{1}\left(S_{h_{n}}\right)}$ converge to $\left\|\varphi_{h_{\infty}}\right\|_{L^{1}\left(S_{h_{\infty}}\right)}$, we have

$$
\left\|\varphi_{h}\right\|_{L^{1}\left(S_{h}\right)} \leq\left\|\varphi_{h_{\infty}}\right\|_{L^{1}\left(S_{h_{\infty}}\right)}
$$

for every $\left(h, S_{h}\right) \in \mathfrak{F}(S, E)$. Hence, $\left(h_{\infty}, S_{h_{\infty}}\right) \in \mathfrak{F}(S, E)$ is extremal for $(S, E, \varphi)$

$$
M_{h_{\infty}}=\sup _{\left(h, S_{h}\right) \in \mathfrak{F}(S, E)} M_{h}
$$

Next we assume that an element $\left(u, S_{u}\right) \in \mathfrak{F}(S, E)$ also attains the supremum. Set

$$
\begin{aligned}
w_{n} & =h_{n} \circ u^{-1}, & \psi_{n} & =\left(\varphi_{h_{n}} \circ w_{n}\right)\left(w_{n}^{\prime}\right)^{2}(n \in \mathbb{N}) \\
w_{\infty} & =h_{\infty} \circ u^{-1}, & \psi_{\infty} & =\left(\varphi_{h_{\infty}} \circ w_{\infty}\right)\left(w_{\infty}^{\prime}\right)^{2} .
\end{aligned}
$$

We show that $\varphi_{u}=\psi_{\infty}$ on $u(S \backslash E)$. For the sake of convenience, we extend $\psi_{n}$ to $S_{u}$ so that $\psi_{n}=0$ on $S_{u} \backslash u\left(U_{n}\right)$ and similarly, $\psi_{\infty}=0$ on $S_{u} \backslash u(S \backslash E)$. Then $\psi_{n}(n \in \mathbb{N})$ and $\psi_{\infty}$ are integrable quadratic differentials on $S_{u}$.

Fix any point $p_{0} \in S_{u}^{\mathrm{d}}$ with $\varphi_{u}^{\mathrm{d}}\left(p_{0}\right) \neq 0$. Let $\zeta_{u}=\xi_{u}+i \eta_{u}$ be the natural parameter of $\varphi_{u}^{\mathrm{d}}$, which is defined about $p_{0}$ by

$$
\zeta_{u}=\int_{z_{0}}^{z} \sqrt{\varphi_{u}^{\mathrm{d}}(z)} d z,
$$

where $z$ is a local chart near $p_{0}$ and $z\left(p_{0}\right)=z_{0}$. Let $N_{p_{0}}$ be a sufficiently small closed neighborhood of $p_{0}$ such that $N_{p_{0}}$ is mapped by $\zeta_{u}$ onto a square centered at $p_{0}$ and each side of which is parallel to the axis. Since $\psi_{n}^{\mathrm{d}}$ is integrable on $S_{u}^{\mathrm{d}}$, the height relative to $\psi_{n}^{\mathrm{d}}$ of almost every vertical segment of $\varphi_{u}^{\mathrm{d}}$ on $S_{u}^{\mathrm{d}}$, particularly on $N_{p_{0}}$, is well defined for each $n \in \mathbb{N} \cup\{\infty\}$.

We denote by $\mathcal{V}_{p_{0}}$ the set of such vertical segments of $\varphi_{u}^{\mathrm{d}}$ in $N_{p_{0}}$.

For each $\beta=\beta\left(\xi_{u}\right) \in \mathcal{V}_{p_{0}}$ we set $\beta_{n}=\beta_{n}\left(\xi_{u}\right)=\beta\left(\xi_{u}\right) \cap u^{\mathrm{d}}\left(U_{n}^{\mathrm{d}}\right)(n \in \mathbb{N})$. Fix any $\varepsilon>0$ and any 1-dimensional measurable set $A \subset N_{p_{0}}$ on a horizontal trajectory of $\varphi_{u}^{\mathrm{d}}$. Since $\varphi^{\mathrm{d}}$ and $\varphi_{u}^{\mathrm{d}}$ are integrable on $S^{\mathrm{d}}$ and $S_{u}^{\mathrm{d}}$, respectively, by Fubini's theorem and Schwarz's inequality, for every $n, m \in \mathbb{N}(n \geq m)$ we have

$$
\begin{aligned}
\int_{A} \text { height }_{\psi_{n}^{\mathrm{d}}}(\beta)-\text { height }_{\psi_{n}^{\mathrm{d}}}\left(\beta_{m}\right) \mid d \xi_{u} & \leq \int_{A} \int_{\beta_{n} \backslash \beta_{m}}\left|\sqrt{\psi_{n}^{\mathrm{d}}\left(\zeta_{u}\right)}\right| d \eta_{u} d \xi_{u} \\
& =\iint_{V(n, m, A)}\left|\sqrt{\psi_{n}^{\mathrm{d}}\left(\zeta_{u}\right)}\right| d \xi_{u} d \eta_{u} \\
& \leq\left\|\psi_{n}^{\mathrm{d}}\right\|_{L^{1}(V(n, m, A))}^{\frac{1}{2}} \cdot\left\|\varphi_{u}^{\mathrm{d}}\right\|_{L^{1}(V(n, m, A))}^{\frac{1}{2}} \\
& \leq\left(c_{1}\left\|\varphi^{\mathrm{d}}\right\|_{L^{1}\left(S^{\mathrm{d}}\right)}\right)^{\frac{1}{2}} \cdot\left\|\varphi_{u}^{\mathrm{d}}\right\|_{L^{1}(V(n, m, A))}^{\frac{1}{2}} \\
& \stackrel{m \rightarrow \infty}{\longrightarrow} 0,
\end{aligned}
$$


where $V(n, m, A)=\left\{p \in N_{p_{0}} \cap u^{\mathrm{d}}\left(U_{n}^{\mathrm{d}} \backslash U_{m}^{\mathrm{d}}\right) \mid \operatorname{Re} \zeta_{u}(p) \in A\right\}$. Then there exists a natural number $N_{1}$ such that for every $n \geq N_{1}$

$$
\int_{A} \mid \operatorname{height}_{\psi_{n}^{\mathrm{d}}}(\beta)-\text { height }_{\psi_{n}^{\mathrm{d}}}\left(\beta_{N_{1}}\right) \mid d \xi_{u}<\varepsilon .
$$

Furthermore we can see that

$$
\left.\int_{A}\right|_{\text {height }_{\psi_{n}^{\mathrm{d}}}}\left(\beta_{N_{1}}\right)-\text { height }_{\psi_{\infty}^{\mathrm{d}}}\left(\beta_{N_{1}}\right) \mid d \xi_{u} \rightarrow 0 \quad(n \rightarrow \infty),
$$

because $\psi_{n}^{\mathrm{d}}$ uniformly converge to $\psi_{\infty}^{\mathrm{d}}$ on $N_{p_{0}} \cap u^{\mathrm{d}}\left(U_{N_{1}}^{\mathrm{d}}\right)$. Then, by Fatou's lemma, we have

$$
\begin{aligned}
& \int_{A} \liminf _{n \rightarrow \infty} \operatorname{height}_{\psi_{n}^{\mathrm{d}}}(\beta) d \xi_{u} \\
& \leq \liminf _{n \rightarrow \infty} \int_{A} \text { height }_{\psi_{n}^{\mathrm{d}}}(\beta) d \xi_{u} \\
& \leq \liminf _{n \rightarrow \infty}\left\{\int_{A}\left(\operatorname{height}_{\psi_{n}^{\mathrm{d}}}(\beta)-\operatorname{height}_{\psi_{n}^{\mathrm{d}}}\left(\beta_{N_{1}}\right)\right) d \xi_{u}\right. \\
& \left.+\int_{A}\left(\operatorname{height}_{\psi_{n}^{\mathrm{d}}}\left(\beta_{N_{1}}\right)-\operatorname{height}_{\psi_{\infty}^{\mathrm{d}}}\left(\beta_{N_{1}}\right)\right) d \xi_{u}+\int_{A} \operatorname{height}_{\psi_{\infty}^{\mathrm{d}}}\left(\beta_{N_{1}}\right) d \xi_{u}\right\} \\
& =\liminf _{n \rightarrow \infty} \int_{A}\left(\operatorname{height}_{\psi_{n}^{\mathrm{d}}}(\beta)-\operatorname{height}_{\psi_{n}^{\mathrm{d}}}\left(\beta_{N_{1}}\right)\right) d \xi_{u} \\
& +\lim _{n \rightarrow \infty} \int_{A}\left(\text { height }_{\psi_{n}^{\mathrm{d}}}\left(\beta_{N_{1}}\right)-\text { height }_{\psi_{\infty}^{\mathrm{d}}}\left(\beta_{N_{1}}\right)\right) d \xi_{u}+\int_{A} \operatorname{height}_{\psi_{\infty}^{\mathrm{d}}}\left(\beta_{N_{1}}\right) d \xi_{u} \\
& \leq \varepsilon+\int_{A} \text { height }_{\psi_{\infty}^{\mathrm{d}}}(\beta) d \xi_{u} .
\end{aligned}
$$

Therefore almost every $\beta \in \mathcal{V}_{p_{0}}$ satisfies

$$
\liminf _{n \rightarrow \infty} \text { height }_{\psi_{n}^{\mathrm{d}}}(\beta) \leq \text { height }_{\psi_{\infty}^{\mathrm{d}}}(\beta) .
$$

The same holds for almost every horizontal and vertical segments on $S_{u}^{\mathrm{d}}$. Then we can see that almost every $\varphi_{u}^{\mathrm{d}}$-polygonal curve $\gamma$, which is a union of finitely many horizontal and vertical segments, satisfies

$$
\liminf _{n \rightarrow \infty} \text { height }_{\psi_{n}^{\mathrm{d}}}(\gamma) \leq \text { height }_{\psi_{\infty}^{\mathrm{d}}}(\gamma)
$$

Moreover, for every $n \in \mathbb{N}, S_{h_{n}}^{\mathrm{d}} \backslash w_{n}^{\mathrm{d}}\left(u^{\mathrm{d}}\left(S^{\mathrm{d}} \backslash E_{n}^{\mathrm{d}}\right)\right)$ is an allowable horizontal slit with respect to $\varphi_{h_{n}}^{\mathrm{d}}$, which consists of finitely many components. Then for almost every $\varphi_{u}^{\mathrm{d}}$-polygonal curve $\gamma$ we can obtain from $w_{n}^{\mathrm{d}}(\gamma)$ a simple closed curve $\tilde{w}_{n}^{\mathrm{d}}(\gamma)$ by supplying finitely many horizontal segments of $\varphi_{h_{n}}^{\mathrm{d}}$. Hence we have

$$
\begin{aligned}
\text { height }_{\varphi_{u}^{\mathrm{d}}}[\gamma] & =\text { height }_{\varphi_{h_{n}}^{\mathrm{d}}}\left[\tilde{w}_{n}^{\mathrm{d}}(\gamma)\right] \\
& \leq \text { height }_{\varphi_{h_{n}}^{\mathrm{d}}}\left(\tilde{w}_{n}^{\mathrm{d}}(\gamma)\right) \\
& =\operatorname{height}_{\varphi_{h_{n}}^{\mathrm{d}}}\left(w_{n}^{\mathrm{d}}(\gamma)\right) \\
& =\operatorname{height}_{\psi_{n}^{\mathrm{d}}}(\gamma)
\end{aligned}
$$


for every $n \in \mathbb{N}$. Therefore height $\varphi_{\varphi_{u}^{\mathrm{d}}}[\gamma] \leq \liminf _{n \rightarrow \infty}$ height $_{\psi_{n}^{\mathrm{d}}}(\gamma)$ holds, and we obtain height $\varphi_{\varphi_{u}^{\mathrm{d}}}[\gamma] \leq$ height $_{\psi_{\infty}^{\mathrm{d}}}(\gamma)$ and, in particular, $\left\|\varphi_{u}^{\mathrm{d}}\right\|_{L^{1}\left(S_{u}^{\mathrm{d}}\right)} \leq\left\|\psi_{\infty}^{\mathrm{d}}\right\|_{L^{1}\left(S_{u}^{\mathrm{d}}\right)}$. Then the maximality of $M_{u}^{\infty}=\left\|\varphi_{u}\right\|_{L^{1}\left(S_{u}\right)}$ implies, together with Proposition 2.4 ,

$$
\varphi_{u}^{\mathrm{d}}=\psi_{\infty}^{\mathrm{d}} \text { on } u^{\mathrm{d}}\left(S^{\mathrm{d}} \backslash E^{\mathrm{d}}\right)
$$

Consequently we have

$$
\varphi_{u}=\left(\varphi_{h_{\infty}} \circ w_{\infty}\right)\left(w_{\infty}^{\prime}\right)^{2} \text { on } u(S \backslash E) .
$$

We have proved Theorem 1.4.

\section{Slit mapping theorem of open Riemann surface of finite genus}

Let $R$ be an open Riemann surface of finite genus. As was observed by Bochner, $R$ can be embedded into a compact Riemann surface $S$ of the same genus. Let $w$ be such a conformal embedding of $R$ into $S$. We note the following fact.

Lemma 5.1. $E=S \backslash w(R)$ is allowable in $S$.

Proof. Let $\kappa$ be the genus of $R$. We can take a family $\left\{\alpha_{1}, \beta_{1}, \alpha_{2}, \beta_{2}, \ldots\right.$, $\left.\alpha_{\kappa}, \beta_{\kappa}\right\}$ of simple closed curves on $R$ with common base point at $p_{0} \in R$ such that $R \backslash \cup_{i=1}^{\kappa}\left(\alpha_{i} \cup \beta_{i}\right)$ is connected. Then $D=S \backslash \cup_{i=1}^{\kappa}\left(w\left(\alpha_{i}\right) \cup w\left(\beta_{i}\right)\right)$ is a simply connected subdomain of $S$. Since $E$ is a compact subset of $D, E$ is relatively contractible in $D$, and hence, in $S$. Since $S \backslash E=w(R)$ is connected, $E$ is allowable.

Let $\mathfrak{M}$ be the family of pairs $\left(g, S_{g}\right)$, where $g$ is a conformal map of $R$ into a compact Riemann surface $S_{g}$ of the same genus. For every $\left(g, S_{g}\right) \in \mathfrak{M}$, $g \circ w^{-1}: S \backslash E \hookrightarrow S_{g}$ induces an isomorphism of $\pi_{1}(S)$ onto $\pi_{1}\left(S_{g}\right)$ by Lemma 2.3. We denote by $\tau=\left[S_{g},\left(g \circ w^{-1}\right)_{*}\right]$ the Teichmüller class in $T(S)$. Then for this $\tau \in T(S)$ and for $\varphi \in A(S) \backslash\{0\}$ we obtain the unique element $\varphi_{g} \in$ $A\left(S_{g}\right) \backslash\{0\}$ whose heights on $S_{g}$ are equal to those of $\varphi$ on $S$. Consider the extremal problem to find an element $\left(g, S_{g}\right) \in \mathfrak{M}$ maximizing $\left\|\varphi_{g}\right\|_{L^{1}\left(S_{g}\right)}$. By Theorem 1.3, we obtain an extremal element $\left(g, S_{g}\right) \in \mathfrak{M}$. The set $S_{g} \backslash g(R)$ is of zero area and each component of $S_{g} \backslash g(R)$ is either

(i) a horizontal arc of $\varphi_{g}$ or,

(ii) a connected union of finitely many horizontal arcs and critical points of $\varphi_{g}$.

Thus we have shown the following

Corollary 5.2. $\quad$ Let $R$ be an open Riemann surface of finite genus. Then there exist a compact Riemann surface $S$ of the same genus and a conformal embedding $g: R \rightarrow S$ and a holomorphic quadratic differential $\psi$ on $S$ such that $S \backslash g(R)$ has measure zero and each component of $S \backslash g(R)$ is a possibly branched arc on horizontal trajectories of $\psi$. 
Acknowledgement. The author wishes to express her deepest gratitude to Professors Takehiko Morita, Masakazu Shiba, Mitsuhiro Shishikura, Toshiyuki Sugawa and Masahiko Taniguchi for many valuable suggestions and ceaseless encouragement. She also thanks the referee who pointed out several errors in the earlier manuscript and advised her.

\author{
Department of Mathematics \\ Graduate School of Science \\ HIROSHIMA UNIVERSITY \\ HigASHI-HiROSHIMA 739-8526, JAPAN \\ e-mail: sasair@jeans.ocn.ne.jp
}

\title{
References
}

[1] L. V. Ahlfors and L. Sario, Riemann Surfaces, Princeton, New Jersey Princeton University Press, 1960.

[2] L. V. Ahlfors, Lectures on Quasiconformal Mappings, Van Nostrand Reinhold, Princeton, 1966.

[3] R. Fehlmann and F. P. Gardiner, Extremal Problem for Quadratic Differentials, Michigan Math. J. 42 (1995), 573-591.

[4] F. P. Gardiner, Teichmüller Theory and Quadratic differentials, Wiley Interscience, 1987.

[5] F. P. Gardiner and N. Lakic, Quasiconformal Teichmüller Theory, Amer. Math. Soc., 2000.

[6] Y. Imayoshi and M. Taniguchi, An Introduction to Teichmüller Space, Springer-Verlag, Tokyo, 1992.

[7] C. Maclachlan and W. J. Harvey, On mapping-class groups and Teichmüller Spase, Proc. London Math. Soc. (3) 30 (1975), 496-512.

[8] L. Sario and M. Nakai, Classification Theory of Riemann Surfaces, Springer-Verlag, Berlin Heidelberg, 1970.

[9] R. Sasai, Non-uniqueness of obstacle problem on finite Riemann surface, preprint.

[10] M. Shiba, The Riemann-Hurwitz relation, parallel slit covering map, and continuation of an open Riemann surface of finite genus, Hiroshima Math. J. 14 (1984), 371-399.

[11] K. Strebel, Quadratic Differentials, Springer-Verlag, Berlin Heidelberg, New York, Tokyo, 1984. 